\title{
Incidence du régime alimentaire et du mode de vie sur les affections systémiques et oculaires ${ }^{2008}$
}

PAR LARRY J ALEXANDER, OD, FAA0

\section{Introduction générale et importance du régime alimentaire}

$\mathrm{F}$ ort d'une pratique de plusieurs années dans les soins de santé, après avoir constaté une augmentation des maladies des complexes immuns, des troubles inflammatoires et des affections dégénératives, j'estime qu'il est temps que le milieu des soins de santé amorce une analyse interactive - Cross-Talk ${ }^{\mathrm{MC}}$. D’après une étude récente, un mode vie sain combinant l'abandon de la cigarette, le maintien d'un poids santé, un régime équilibré, la consommation modérée d'alcool et l'exercice quotidien réduirait les accidents ischémiques cérébraux de près de moitié chez les hommes comme chez les femmes ${ }^{1}$. La communication de cette information essentielle auprès de tous les fournisseurs de soins de santé est importante pour assurer la santé à long terme de la population. L'initiative Cross-Talk ${ }^{\mathrm{MC}}$ s'inscrit dans cet ordre d'idées.

La plupart des spécialisations et des sous-spécialisations ont leurs revues dans lesquelles sont publiées leurs découvertes sans que les conclusions ne soient communiquées aux autres branches de la médecine, et ce, même si elles pouvaient avoir une incidence directe sur la gestion d'autres systèmes et appareils de l'organisme. À titre d'exemple, combien de praticiens traitant les cas de zona ont lu l'article suivant?

Herpes zoster. The treatment and prevention of neuralgia with adenosine monophosphate. ${ }^{2}$

(Zona : le traitement et la prévention de la névralgie par l'adénosine monophosphate)

[Traduction] Trente-deux adultes ont pris part à un essai aléatoire à double insu, avec groupe témoin, qui consistait en des injections intramusculaires d'adénosine monophosphate (AMP) en gel, trois fois par semaine pendant quatre semaines, pour traiter le zona. L'AMP a entraîné une diminution modérée de la douleur peu après le début du traitement, en plus de réduire la période de desquamation et de favoriser une guérison plus rapide de la peau que dans le groupe témoin. Le traitement à l'AMP a réduit l'excrétion du virus et éliminé le virus plus rapidement que dans le groupe témoin. Au terme de la première période de traitement de quatre semaines, $88 \%$ des patients traités à l'AMP n'éprouvaient plus de douleur, par opposition à seulement $43 \%$ de ceux du groupe témoin. Après quatre semaines, tous les patients éprouvant encore de la douleur ont commencé à recevoir un traitement à l'AMP sans infraction au code. Aucun ne ressentait encore de la douleur dans les trois semaines suivant le début du traitement. Ils n’ont connu aucune récurrence de la douleur ou des lésions pendant trois à dix-huit mois après la fin du traitement. Ni effet secondaire ni phénomène de toxicité n'ont été associés à l'AMP, un métabolite cellulaire naturel, durant et après le traitement.

L'œil est tout désigné pour illustrer l'incidence que peut exercer une spécialisation sur une autre. Ainsi, les cardiologues sont bien conscients du rôle possible de certains suppléments nutritifs dans la gestion des troubles vasculaires. Une modification oculaire d'origine vasculaire peut entrainer un certain nombre de troubles, comme le glaucome et la dégénérescence maculaire. Il y a donc lieu de se demander pourquoi les professionnels des soins oculaires ne font pas une incursion du côté de la cardiologie dans leur réflexion. En dermatologie, on s'est aperçu que les niveaux de coenzyme Q10 (CoQ10) indiquaient la probabilité d'une progression éventuelle du mélanome malin, l'une des affections les plus redoutées pour la santé des yeux.

Il est particulièrement intéressant de s'arrêter à l'influence du contexte social sur le plan comportemental en ce qui a trait à l'apparition de déficiences nutritionnelles. La pauvreté, les effets secondaires des médicaments, l'abus de médicaments, l'alcoolisme, le pontage gastrique (chirurgie bariatrique), une alimentation riche en matières grasses et la simple ignorance peuvent ouvrir

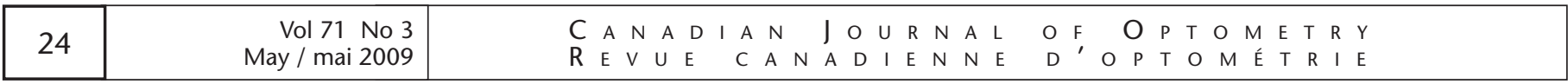


la voie à une foule de maladies et de troubles. Aussi devons-nous intégrer les problèmes d'origine alimentaire à notre réflexion sur le développement des maladies oculaires. ${ }^{3} \mathrm{La}$ diminution de la survie des participants à l'étude AREDS (Age-Related Eye Disease Study) qui étaient atteints d'une dégénérescence maculaire liée à l'âge (DMA) et de cataractes permet de penser que ces affections reflétaient davantage un état systémique que des processus uniquement locaux. ${ }^{4} \mathrm{La}$ carence en vitamine A peut à tout le moins être associée à une malabsorption de matières grasses, elle-même peut-être liée à une chirurgie bariatrique $^{5}$ qui influera ensuite directement sur la genèse du syndrome de l'œil sec. La carence en vitamine B12 et en acide folique est également associée à un problème de malabsorption, en plus d'être connue pour ses effets secondaires oculaires fortement liés à l'hyperhomocystéinémie, qui représente une importante menace cardiovasculaire. ${ }^{6-9}$ En dépit d'un régime prétendument sain, de $10 \%$ à $14 \%$ des Américains accusent une carence en vitamine $\mathrm{C}^{10}$, et une carence en vitamine B12 a été prouvée en laboratoire chez jusqu'à $15 \%$ des adultes âgés de plus de 60 $a_{n}{ }^{6}$. Le traitement de la majorité des patients au moyen de ces nutriments aisément accessibles suppose de bons choix alimentaires ou la consommation de suppléments ainsi que de l'exercice et une modification générale des comportements, notamment l'abandon de la cigarette et la réduction de la consommation d'alcool. Il est toujours étonnant de voir que les patients croient encore à l'existence d'une cure miracle. Une récente étude montre que, malgré une alimentation riche en acides gras oméga-3, les patients inuits de l'Alaska sont aux prises avec une athérosclérose infraclinique à un jeune âge, probablement imputable, en grande partie, à un usage excessif du tabac ${ }^{11}$. L'idée centrale tient à l'importance de la synergie, plus valable qu'une approche axée sur une monothérapie, pour la gestion de la plupart des affections chroniques, neurodégénératives et inflammatoires. La « modulation » (équilibre) est le maitre mot pour améliorer l'état de santé et réduire les risques. Rappelons que les changements comportementaux radicaux et les thérapies déséquilibrées peuvent créer plus de tort que de bien. La combinaison de l'exercice et de thérapies est à l'origine de récents « eurêkas ». De fait, si l'exercice régulier et la consommation d'acides gras oméga-3 à longue chaîne, tirés du poisson ou de l'huile de poisson, peuvent à eux seuls améliorer la santé cardiovasculaire et métabolique, leur combinaison s'avère plus efficace (American Journal of Clinical Nutrition, 2007, vol. 85, p. 1267). Il n'existe pas de cure miracle, mais une attitude et un style de vie propices à l'amélioration de la santé s'imposent. Bien que nous nous attendions à ce que chaque patient modifie ses comportements pour augmenter ses chances de connaitre une meilleure santé, nous devons savoir que cela ne se produira pas facilement et que nous serons appelés à devenir des « conseillers personnels » auprès d'eux en matière de santé. Une récente étude corrobore cette conclusion, mentionnant que les modes de vie sains comprennent l'abandon de la cigarette, le maintien d'un bon IMC, une consommation d'alcool modérée et l'exercice quotidien. Chez les hommes et les femmes, l'adoption de modes de vie sains est associée à une réduction de $80 \%$ du risque d'accident vasculaire cérébral ${ }^{1}$.

Le fait qu'un fournisseur de soins de santé ignore souvent la mesure dans laquelle son patient utilise des produits non pharmaceutiques pose une autre question importante au sujet de la consommation de suppléments. Il arrive que des patients utilisant des anticoagulants ne déclarent pas leur consommation de vitamine $\mathrm{E}$ et de gingko biloba. La consommation de nombreux suppléments aura une incidence certaine sur les temps de prothrombine, entraînant même une capacité accrue de saigner. D’après une enquête réalisée en 1999, près de $49 \%$ des Américains adultes auraient utilisé des produits à base d'herbes médicinales au cours de l'année précédente. Or, il a été prouvé que jusqu'à $31 \%$ des patients qui utilisent ces produits les combinent avec des médicaments d'ordonnance, et qu'environ $70 \%$ de ces derniers patients ne déclarent pas de façon régulière la consommation de tels produits à leurs fournisseurs de soins de santé. ${ }^{12-13}$

Un autre rapport instructif fait état $\mathrm{du}$ recours des personnes atteintes de cancer aux médecines douces et autres approches thérapeutiques. La prière et d'autres pratiques spirituelles seraient les approches les plus fréquentes, déclarées par 61,4\% des survivants. Viendraient ensuite la relaxation (44,3\%), la guérison par la foi $(42,4 \%)$, la consommation de suppléments nutritifs et de vitamines $(40,1 \%)$, la méditation (15\%), la consultation religieuse $(11,3 \%)$, les massages $(11,2 \%)$ et la fréquentation de groupes de soutien $(9,7 \%)$. L'hypnose était une méthode moins 
tion s'expose également à un risque accru. ${ }^{27} \mathrm{Un}$ autre rapport récent indique que les facteurs de risque de maladie cardiovasculaire chez les Latino-Américains peuvent influer sur la DMA avancée. ${ }^{28} \mathrm{Il}$ n'y a pas lieu de s'en étonner depuis les premiers travaux de Richer, qui ont démontré que les facteurs de risque de maladie cardiovasculaire, y compris les niveaux de fer sérique, contribuaient à la progression de la DMA. ${ }^{29-30}$ Les autres facteurs de risque souvent associés aux maladies du cœur, comme le tabagisme et les changements à la composition du sang, peuvent aussi être modifiés dans notre base de patients. ${ }^{31-35}$ Des études attestent également du fait que l'obésité est actuellement liée à une dégénérescence pigmentaire de la macula, qui pourrait être attribuable à la concurrence inhérente du tissu adipeux. ${ }^{36-38}$ Les taux sériques de lutéine et de zéaxanthine sont les véritables mesures de l'efficacité des effets de protection du régime alimentaire, et ces deux taux sont inférieurs lorsqu'il y a obésité et diabète. ${ }^{36} \mathrm{Si}$ toutes ces études ne concluent pas de façon absolue au besoin d'une diète d'un point de vue scientifique, elles souli gnent l'importance de l'abandon de la cigarette pour réduire le risque de DMA. ${ }^{39}$

Beaucoup d'analyses ont porté sur le régime alimentaire par rapport au développement des cataractes. Bien qu'elles soient très ciblées, certaines ont établi un lien entre le syndrome métabolique et la genèse des cataractes. $^{40-41}$ Tout indique qu'il existe un lien entre le stress oxydatif et la formation de cataractes si, encore une fois, un problème de tabagisme est en cause. ${ }^{42}$ En général, il s'avère aussi que l'obésité indique une probabilité accrue de développer une cataracte, bien que ce marqueur soit légèrement imprévisible en fonction du type de cataracte. ${ }^{43-45}$ Si l'indice de masse corporelle (IMC), l'obésité abdominale et le diabète sont plus élevés, l'incidence d'une cataracte corticale et d'une cataracte sous-capsulaire postérieure l'est aussi. ${ }^{46-47}$

Le lien entre le diabète et l'obésité (plus particulièrement le syndrome métabolique) est incontestable. ${ }^{48-51}$ Le syndrome métabolique dénote l'existence d'une série de facteurs de risque naturellement reliés, notamment l'obésité, une pression artérielle élevée, une résistance à l'insuline, la dyslipidémie, un état proinflammatoire et un état prothrombotique. Ce scénario (syndrome métabolique) peut entraîner plusieurs problèmes de flux vasculaire rétinien dans l'œil. ${ }^{52}$ Le lien avec la rétinopathie diabétique fait l'objet d'une plus grande circonspection, mais des études ont associé la microvasculopathie rétinienne au syndrome métabolique. ${ }^{49,53}$ L'inhibition des médiateurs inflammatoires intervient aussi dans la réduction des risques de diabète ${ }^{54}$, et des changements au régime alimentaire peuvent la favoriser. Une diète axée sur le syndrome métabolique peut constituer la solution à explorer pour réduire le risque de rétinopathie diabétique, mais les essais cliniques doivent corroborer cette conclusion. De plus, il importe de se pencher sur d'autres facteurs qui peuvent accroître le stress oxydatif et diminuer l'approvisionnement de l'œil en sang oxygéné, comme le tabagisme et l'apnée du sommeil.

Il y a également une association à faire entre les problèmes de poids et le glaucome. Il ressort sans équivoque de certaines études qu'un lien existe entre, d'une part, la résistance à l'insuline et le syndrome métabolique et, d'autre part, la pression intraoculaire. ${ }^{55}$ L'indice de masse corporelle semble lié à une pression intraoculaire élevée. ${ }^{56-59} \mathrm{Il}$ va sans dire que la première réaction des cliniciens face à un tel fait serait d'examiner la taille du cou ainsi que la pression positive pour conclure qu'il s'agit de facteurs associés à l'apnée du sommeil, qui correspondent à cette analyse. $^{60-61}$ La relation entre l'élévation de la pression du liquide céphalorachidien, l'hypertension intracrânienne idiopathique, les concentrations sériques de cortisol et l'apnée du sommeil forme un scénario digne d'intérêt en ce qui concerne la pression intraoculaire élevée. ${ }^{62}$ Bien qu'une analyse approfondie d'un point de vue scientifique s'impose, il semble, assez logiquement, que l'obésité ait un lien avec le glaucome, si celui-ci n'est pas imputable à un autre état physique restreint.

Cordain soutient que le régime alimentaire occidental a évolué d'une façon qui ne répond pas à nos besoins biologiques de base. ${ }^{63}$ Cette affirmation s'appuie sur l'idée que nous possédons encore les gènes de l'homme des cavernes (génotype), lesquels exigent une alimentation de type paléolithique qui ne correspond pas à notre régime alimentaire actuel. Celui-ci, jugé aberrant, créerait un stress oxydatif qui influerait à la fois sur les réactions inflammatoires et le système immunitaire. Dans le cas d'un glaucome, le stress oxydatif mène à des modifications aux cellules ganglionnaires de la rétine, qui précipitent la progression des 
dommages. L'obtention de résultats similaires est vraisemblable pour d'autres troubles oculaires. Sans une conversion appropriée de ces radicaux, le stress empoisonnerait le système. ${ }^{64}$ Les produits phytochimiques, tels le thé vert ou noir ${ }^{65-67}$, le café ${ }^{68}$, le chocolat noir ${ }^{69}$ et le vin rouge $^{70-71}$, contiennent des composés polyphénoliques qui agissent comme des phagocytes de radicaux libres. ${ }^{72}$ Selon un récent article axé sur un sous-groupe de femmes noires, la consommation de trois portions ou plus de fruits par jour entrainerait une diminution de $79 \%$ du risque de glaucome, par comparaison à celles en consommant moins d'une par jour. ${ }^{73}$ Une autre étude portant sur des femmes afro-américaines conclut, comme on pouvait s'y attendre, à l'existence d'un lien entre, d'une part, une consommation plus élevée de boissons gazeuses et de boissons aux fruits et, d'autre part, une incidence accrue de diabète de type 2. ${ }^{74}$ L'équilibre (MODULATION) est le mot d'ordre en ce qui a trait à l'alimentation en général, à la consommation de suppléments et à la modification des comportements.

Par ailleurs, une récente étude présente les recommandations suivantes pour adopter un régime antiinflammatoire susceptible d'améliorer l'état de santé général et, de façon plus particulière, le système cardiovasculaire, dont l'incidence sur les troubles oculaires n'est plus à démontrer, ni le risque de développer des maladies neurodégénératives qui en découle. Très bons indicateurs de la présence d'une inflammation systémique, les niveaux de protéines $C$ réactives seraient élevés dans de nombreux troubles oculaires.
Un régime anti-inflammatoire peut s'avérer profitable à toute personne souffrant d'une affection accompagnée d'un nombre élevé de protéines $\mathrm{C}$ réactives.

"Ce régime anti-inflammatoire doit être considéré comme le moyen de prévention principal ou secondaire de la coronaropathie et du diabète. ${ }^{75}$ »

L'indice glycémique d'un aliment est défini comme la surface incrémentielle se trouvant sous la courbe de glucose postprandial associée à l'ingestion d'une portion de $50 \mathrm{~g}$ d'un aliment particulier, par opposition à celle de $50 \mathrm{~g}$ de glucose par voie orale. Les glucides idéaux, c'està-dire ceux dont l'indice glycémique est faible, comprennent les légumes à feuilles vertes, comme le brocoli et l'épinard, et les fruits, tels le pamplemousse et les cerises. Optez pour des glucides à haute teneur en fibres et dont l'indice glycémique est faible - légumes, fruits, grains entiers, légumineuses et noix.

Une consommation excessive de glucides transformés provoque un cycle vicieux de pics transitoires dans les niveaux de glycémie, en plus d'entraîner une augmentation de la production d'insuline et de l'hypoglycémie réactive. Évitez les aliments et les boissons très transformés, plus particulièrement ceux contenant du sucre, du sirop de glucose à haute teneur en fructose, de la farine blanche ou des gras trans.

Les petits fruits, le chocolat noir, le vin rouge, le thé et les grenades réduisent le stress oxydant postprandial ainsi que l'inflammation. Les fèves de cacao contiennent une sous-catégorie de flavonoïdes dont on a prouvé la capacité à aug- menter la quantité d'eNOS et, par le fait même, de NO. Cette propriété améliore la vasorelaxation d'origine endothéliale. $^{76}$ D'après une étude, un carré de chocolat noir comporte 6,3 g et ne représente que $30 \mathrm{kcal}$ par jour, et des études antérieures ont montré que $100 \mathrm{~g}$ de chocolat noir abaissent la pression artérielle de $12 / 9 \mathrm{~mm} \mathrm{Hg}$, mais risquent d'accroitre l'apport calorique. ${ }^{77}$

Le café contient des antioxydants et peut améliorer la sensibilité à l'insuline. La consommation de thé noir réduit l'activation plaquettaire et la concentration plasmique de protéines $\mathrm{C}$ réactives. Cependant, une recherche antérieure n'a pas conclu à une réduction constante du risque d'accident vasculaire cérébral associé à la consommation de café ou de thé. Une étude tend à indiquer qu'une consommation plus élevée de café et de thé peut réduire le risque d'infarctus cérébral chez les hommes qui fument, mais pas celui d'hémorragie intracrânienne. $^{78}$

Ajoutée à un repas dont l'indice glycémique est élevé, la cannelle ralentit la vidange gastrique et réduit l'excursion de glucose postprandial.

Les noix ralentissent également la vidange gastrique et peuvent réduire de moitié l'effet des glucides dont l'indice glycémique est élevé. Elles diminuent également les dommages imputables aux protéines oxydatives postprandiales. La consommation de noix au moins cinq fois par semaine peut faire baisser les risques de coronaropathie et le diabète dans une proportion de $20 \%$ à $50 \%$. Mangez environ une poignée de noix chaque jour (un poing fermé), et accompagnez-les de légumes, de grains, de petits fruits ou d'autres fruits. 
Le vinaigre peut réduire la glycémie postprandiale, en plus d'accroître la satiété. Mangez chaque jour de la salade de feuilles vertes, relevée d'une vinaigrette composée de vinaigre et d'huile d'olive.

Les protéines maigres réduisent l'excursion du glucose postprandial et améliorent la satiété. Ces protéines se trouvent dans les blancs d'œuf, la viande de gibier, la poitrine de volaille sans la peau et les protéines de lactosérum ou toute source de protéines laitières non grasses. Consommez des protéines maigres au cours des trois repas de la journée.

Le fait de consommer une boisson alcoolisée $(0,5 \%$ à $1 \%$ ) par jour chez les femmes et d'une à deux boissons alcoolisées par jour chez les hommes peut réduire le risque de maladie cardiovasculaire. La consommation d'une à deux boissons avant un repas peut réduire les taux de glucose postprandial et d'insuline. Cependant, des quantités plus élevées peuvent nuire au métabolisme du glucose.
Selon le niveau d'intensité, l'exercice abaisse les taux de glucose et de triglycérides. Faites de l'activité physique au moins 30 minutes par jour, à une intensité au moins modérée.

Maintenez un poids normal et évitez de vous trouver en surpoids ou dans un état d'obésité. La circonférence de la taille ne devrait jamais dépasser la moitié de la grandeur d'une personne.

Il a été prouvé qu'un régime composé d'aliments à faible indice glycémique était propice à la perte de poids et à l'amélioration du profil lipidique. ${ }^{79}$ De plus, les femmes du quintile le plus élevé d'un groupe dont le régime était composé d'aliments à forte teneur en lipides et à faible teneur en fibres s'exposaient à un risque relativement plus élevé de développer un cancer du colon $(1,46)$, comparativement à celles du quintile inférieur. En revanche, la consommation d'aliments à haute teneur en fibres et de protéines saines

\section{CARACTÉRISTIQUES DU SYNDROME MÉTABOLIQUE :}

1. Obésité abdominale;

2. Dyslipidémie athérogène;

3. Pression artérielle élevée;

4. Taux d'insuline élevés - plus de 10 :

a. Augmentation des matières grasses dans les cellules;

b. Accroissement du stockage de gras;

c. Stimulation des cellules des muscles lisses de la paroi vasculaire;

d. Hausse de la production de mauvais

types d'éicosanoïdes (EC) - hormones

intracellulaires;

e. Les EC de type 1, bons, risquent d'être inhibés par une trop grande

quantité de graines de lin;

f. Les EC de type 2, de mauvais gluca-

gons, sont d'importants inhibiteurs;

5. Rétention rénale des liquides

Le glucagon agit comme anti-insuline,

et les aliments à haute teneur en

protéines et à faible teneur en glucides

le stimulent.

6. Production d'une quantité élevée de médiateurs inflammatoires, mesurée en fonction des taux de protéines

réactives $C$. est associée à une réduction des taux de cancer du colon. Le régime alimentaire n'aurait pas une forte incidence sur le risque de cancer rectal ${ }^{80}$.

Idéalement, nous serions tous des adeptes du régime paléolithique et de l'exercice, nous maintiendrions un poids sain et nous ne consommerions pas de substances potentiellement toxiques. L'utilisation de suppléments serait alors totalement futile. Cependant, il y a loin de la coupe aux lèvres, et même les personnes les plus exemplaires sombrent parfois dans un mode de vie qui laisse à désirer, d'où l'importance des rappels, notamment pour la consommation de suppléments. Il est également essentiel, par rapport à toutes ces considérations, que nous tenions compte des toxicités et des interactions éventuelles.

\section{La deuxième partie de cet article} portera sur les avantages déclarés de certains nutriments et suppléments.

Le $D^{r}$ Alexander ne touche aucune compensation auprès d'entreprises spécialisées dans les suppléments nutritifs. Il agit comme conseiller non rémunéré auprès de Biosyntrx. Il dirige une entreprise d'imagerie numérique, Clinical Education for Optovue Inc., qui produit le RTV ue.

\section{Références}

1. D. Lie, «Overall healthy lifestyle significantly reduces stroke risk », Medscapte CME, 15 août 2008, en ligne le 11 août 2008.

2. S. H. Sklar, W. T. Blue, J.E. Alexander et coll. « Herpes zoster. The treatment and prevention of neuralgia with adenosine monophosphate ", Journal of the American Medical Association, 1985, vol. 253, n 10 , p. $1427-1430$. 
3. G. Kullman, J.L. Bennett, N. Mandava et M. Kahook, «Nutritional Deficiencies », Glaucoma Today, 2007, no 5, p. 33-35.

4. «AREDS Report No. 13: AREDS Research Group associations of mortality with ocular disorders and an intervention of high-dose antioxidants and zinc in the Age-Related Eye Disease Study ", Archives of Ophthalmology, 2004, $\mathrm{n}^{\circ}$ 122, p. 716-726.

5. Y. Spits, J.J. De Laey et B.P. Leroy, «Rapid recovery of night blindness due to obesity surgery after vitamin A repletion therapy ", British Journal of Ophthalmology, 2004, vol. 88, no 4, p. 583585.

6. E. Andràs, S. Affenberger, S. Vinzio et coll. «Food-cobalimin malabsorption in elderly patients: clinical manifestations and treatment », American Journal of Medicine, 2005, vol. 118, p. 1154-1159.

7. A. Hvas et E. Nexo, «Diagnosis and treatment of vitamin $B_{12}$ deficiency-an update », Haematologica, 2006, vol. 91, p. 1506-1512.

8. A. A. Sadun, « Metabolic optic neuropathies ", Semin Ophthalmol, 2002, vol. 17, p. 29-32.

9. C.M. Pfeiffer, S.P. Caudill, E.W. Gunter et coll., « Biochemical indicators of $B$ vitamin status in the US population after folic acid fortification: results from the National Health and Nutrition Examination Survey 1999-2000», American Journal of Clinical Nutrition, 2005, vol. 82, p. 442-450.

10. J.S. Hampl, C.A. Taylor et C.S. Johnston, "Vitamin C deficiency and depletion in the United States: the Third National Health and Nutrition Examination Survey, 1988 to 1994 », American Journal of Public Health, 2004, vol. 94, p. 870-875.

11. A. Cutchins, M.J. Roman, R.B. Devereux et coll. « Prevalence and correlates of sub-clinical atherosclerosis in Alaska Eskimos: The GOCADAN study ", Stroke, 2008 (DOI:10.1161/ STROKEAHA.108.519199), accessible en ligne à stroke.ahajournals.org.

12. J.H. Wirth, J.C. Hudgins et J.A. Paice, "Use of herbal therapies to relieve pain: A review of efficacy and adverse effects », Pain Management Nursing, 2005, vol. 6, p.145-167.

13. W. Abebe, «Herbal medication: Potential for adverse interactions with analgesic drugs ", Journal of Clinical Pharmacy \& Therapentics, 2002, vol. 27, p. 391-401.

14. T. Gansler, C. Kaw, C. Crammer et T. Smith, «A population-based study of prevalence of complementary methods use by cancer survivors: a report from the American Cancer Society's studies of cancer survivors ", Cancer, 2008, vol.113, p.1048-1057.

15. V.M. Montori, P.J. Devereaux, N.K. Adhikari et coll., « Randomized trials stopped early for benefit: a systematic review ", Journal of the American Medical Association, 2005, vol. 294, p. 2203-2209.

16. J.P. Ioannidis, « Contradicted and initially stronger effects in highly cited clinical research ", Journal of the American Medical Association, 2005, vol. 294, p. 218-228.

17. J.P. Ioannidis, «Limitations are not properly acknowledged in the scientific literature ", Journal of Clinical Epidemiology, 2007, vol. 60, p. 324-329.

18. N.A. Trikalinos, E. Evangelou et J.P. Ioannidis, «Falsified papers in highimpact journals were slow to retract and indistinguishable from nonfraudulent papers ", Journal of Clinical Epidemiology, 2008, vol. 61, p. 464-470.

19. J.P. Ioannidis, «Effect of the Statistical Significance of Results on the Time to Completion and Publication of Randomized Efficacy Trials », Journal of the American Medical Association, 1998, vol. 279 , p. 281-286.

20. J.P. Ioannidis et T.A. Trikalinos, «An exploratory test for an excess of significant findings ", Clinical Trials, 2007, vol. 4, p. 245-253.

21. Programme et résumés de la réunion annuelle de l'Association for Research in Vision and Ophthalmology, du 27 avril au $1^{\text {er }}$ mai 2008, Fort Lauderdale, Floride (résumé 1219).

22. N. Cheung et T.Y. Wong, "Obesity and eye diseases », Survey of Ophthalmology, 2007, vol. 52, p. 180-95.

23. A.V. Everitt, S.N. Hilmer, J.C. BrandMiller et coll., «Dietary approaches that delay Age-Related diseases », Clinical Interventions in Aging, 2006, vol. 1, p.1131.

24. L.P. Santos, J.R. Dinez, A.C. Leao et M.F. Sena, «Degeneração macular relacionada à idade: prevalência e fatores de risco em dois centros oftalmológicos de referência em Pernambuco », Arquivos brasileiros de oftalmologia, 2005, vol. 68, p. 229-233.

25. J.M. Seddon, J. Cote, N. Davis et B. Rosner, « Progression of Age-Related macular degeneration ", Archives of Ophthalmology, 2003, vol. 121, p. 785-792.

26. H.A. Moeini, H. Masoudpour et H. Ghanbari, «A study of the relation between body mass index and the incidence of Age-Related macular degeneration », British Journal of Ophthalmology, 2005, vol. 89, p. 964-966.

27. D.A. Schaumberg, W.G. Christen, S.E. Hankinson et R.J. Glynn, « Body mass index and the incidence of visually significant Age-Related maculopathy in men ", Archives of Ophthalmology, 2001, vol. 119, p. 1259-1264.

28. S. Fraser-Bell, J. Wu, R. Klein et coll., «Cardiovascular risk factors and agerelated macular degeneration: the Los Angeles Latino Eye Study ", American Journal of Ophtalmology, 2008, vol. 145, p. 308-316.

29. S. Richer, D. Rudy, L. Statkute et coll., «Serum iron, transferrin saturation, ferritin, and dietary data in age-related macular degeneration ", American Journal of Therapentics, janvier-février 2002, vol. 9 , no 1, p. 25-28.

30. S. Richer, J. Devenport et J.C. Lang, «LAST II: Differential temporal responses of macular pigment optical density in patients with atrophic agerelated macular degeneration to dietary supplementation with xanthophylls ", Optometry, 2007, vol. 78, p. 213-219.

31. T.E. Clemons, R.C. Milton, R. Klein, J.M. Seddon et F.L. Ferris, "The Age Related Eye Disease Study Group. Risk factors for the incidence of advanced Age-Related Macular degeneration in the Age-Related Eye Disease Study (AREDS) » (rapport n'19 de l'AREDS), Ophthalmology, 2005, vol. 112, p. 533-539.

32. R. Klein, Y. Deng, B.E.K. Klein et coll., «Cardiovascular disease, its risk factors and treatment, and age-related macular degeneration: Women's health initiative sight exam ancillary study ", American Journal of Ophtalmology, 2007, vol. 143, p. 473-483.

33. D.A. Schaumberg, S.E. Hankinson, Q. Guo et coll., «A prospective study of 2 major Age-Related macular degeneration susceptibility alleles

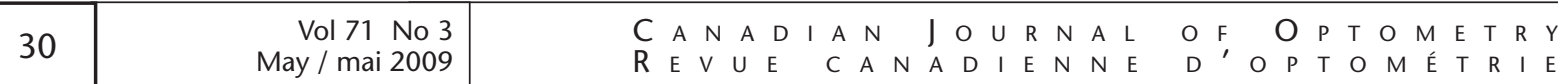


and interactions with modifiable risk factors ", Archives of Ophthalmology, 2007, vol. 125 , p. 55-62.

34. W. Smith, P. Mitchell, S.R. Leeder et J.J. Wang, « Plasma fibrinogen Levels, other cardiovascular risk factors, and Age-Related maculopathy ", Archives of Ophthalmology, 1998, vol. 116, p. 583-587.

35. M. Drobek-Slowik, D. Karczewicz et K. Safranow, «The potential role of oxidative stress in the pathogenesis of the age-related macular degeneration (AMD) », Postepy Hig Med Dose, 2007, vol. 61, p. 28-37.

36. J.A. Mares, T.L. Rowe, D.M. Snoddery et coll., «CAREDS Macular Pigment Study Group and Investigators. Predictors of optical density of lutein and zeaxanthin in retinas of older women in the Carotenoids in Age-Related Eye Disease Study, an ancillary study of the Women's Health Initiative ", American Journal of Clinical Nutrition, 2006, vol. 84, p. 1107 1122.

37.E.J. Johnson, « Obesity, Lutein metabolism, and Age-Related Macular degeneration: A Web of Connections ", Nutrition Reviews, 2005, vol. 63, p. 9-15.

38. B.R. Hammond, T.A. Ciulla et M. Snodderly, « Macular pigment density is reduced in obese subjects ", Investigative Ophthalmology \& Visual Science, 2002, vol. 43, p. 47-50.

39. R.H. Guymer et E.W. Chong, «Modifiable risk factors for Age-Related macular degeneration ", Medical Journal of Australia, 2006, vol. 184, p. 455-458.

40. F. Bojarskiene, L.R. Cerniauskiene, A. Paunksnis et D.I. Luksiene, «Association of metabolic syndrome components with cataract », Medicina (Kaunas), 2006, vol. 42, p. 115-122.

41. A. Paunksnis, F. Bojarskiene, A. Cimbalas et coll., « Relation between cataract and metabolic syndrome and its components ", European Journal of Ophtalmology, 2007, vol. 17, n 4, p. 605614.

42. H. Shichi, « Cataract formation and prevention ", Expert opinion on investigational drugs, 2004, vol. 13, p. 691701.

43. A. Paunksnis, F. Bojarskiene, A. Cimbalas et coll., « Relation between cataract and metabolic syndrome and Its components », European Journal of
Ophtalmology, 2007, vol. 17, p. 605-614.

44. E. Navarro, L. Butierrez, C. Valero et coll., « Prevalence and risk factors of Lens Opacities in the Elderly in Cuenca, Spain ", European Journal of Ophtalmology, 2007, vol. 17, p. 29-37.

45. T.M. Kuang, SY Tsai, W.M. Hsu et coll., «Body mass index and Age-Related cataract; the Shihpai Eye Study », Archives of Ophthalmology, 2005, vol. 123, p. 1109-1114.

46. R. Hiller, M.J. Podgor, R.D. Sperduto et coll., «A longitudinal study of body mass index and lens opacities. The Framingham Studies ", Ophthalmology, 1998, vol. 105, p. 1244-1250.

47. P.F. Jacques, S.M. Moeller, S.E. Hankinson et coll., «Weight status, abdominal adiposity, diabetes, and Early Age-Related lens opacities ", American Journal of Clinical Nutrition, 2003, vol. 78, p. 400-405.

48. D.L. Burnet, L.D. Elliott, M.T. Quinn et coll., " Preventing diabetes in the clinical setting », Journal of General Internal Medicine, 2006, vol. 21, p. 84-93.

49. B.E. Klein, R. Klein et K.E. Lee, «Components of the metabolic syndrome and risk of cardiovascular disease and diabetes in Beaver Dam ", Diabetes Care, 2002, vol. 25, p. 1790 1794.

50. M. B. Weber et K.M. Narayan, «Preventing Type 2 Diabetes: Genes or Lifestyle? ", Primary care diabetes, 2008, vol. 2, p. 65-66.

51. S.M. Twigg, M.C. Kamp, T.M. Davis et coll., « Prediabetes: A Position Statement From the Australian Diabetes Society and Australian Diabetes Educators Association ", Medical Journal of Australia, 2007, vol. 186, p. 461-465.

52. N. Kosanovic-Jakovic, B. Ivanovic, S. Milenkovic et coll., «Anterior ischemic optic neuropathy associated with metabolic syndrome ", Arquivos brasileiros de oftalmologia, 2008, vol. 71, p. 62-66.

53. T.Y. Wong, F.M. Islam, R. Klein et coll., « Retinal vascular caliber, cardiovascular risk factors, and inflammation: The Multi-Ethnic Study of Atherosclerosis (MESA) ", Investigative Opbthalmology \& Visual Science, 2006, vol. 47, p. 2341 2350.

54. T.S. Kern, « Contributions of inflammatory processes to the development of the early stages of diabetic retinopathy ", Experimental Diabets Research, 2007, vol. 2007, 95103.

55. S.W.Oh, S. Lee, C. Park et D.J. Kim, «Elevated intraocular pressure is associated with insulin resistance and metabolic syndrome, Diabetes/Metabolism Research and Reviews, 2005, vol. 21, p. 434 440.

56. M. Yoshida, M. Ishikawa, A. Kokaze, et coll. «Association of Life-Style with intraocular pressure in Middle-Aged and older Japanese residents ", Japanese journal of ophthalmology, 2003, vol. 47, p.191-198.

57. K. Mori, F. Ando, H. Nomura et coll., "Relationship between intraocular pressure and obesity in Japan ", International Journal of Epidemiology, 2000, vol. 29, p. 661-666.

58. J.S. Lee, S.H. Lee, B.S. Oum et coll., «Relationship between intraocular pressure and systemic health parameters in a Korean population », Clinical \& experimental ophthalmology, 2002, vol. 30, p. 237-241.

59. F. Memarzadeh, M. Ying-Lai, S.P. Azen et coll., «Associations with intraocular pressure in Latinos: the Los Angeles Latino Eye Study ", American Journal of Ophtalmology, 2008, vol. 146, p. 69-76.

60. A.A. McNab, «The eye and sleep apnea », Sleep medicine reviews, 2007, vol. 11, p. 269-276.

61. S. Dhillon, C.M. Shapiro et J. Flanagan, «Sleep-Disordered breathing and effects on ocular health ", Journal canadien d'ophtalmologie, 2007, vol. 42, p. 238-243.

62. A.B. Daniels, G.T. Liu, N.J. Volpe et coll., «Profiles of obesity, weight gain, and quality of life in idiopathic intracranial hypertension (pseudotumor cerebri) ", American Journal of Ophtalmology, 2007, vol. 143, p. 635-641.

63. L. Cordain, S.B. Eaton, A. Sebastian et coll., "Origins and evolution of the western diet: health implications for the 21st century ", American Journal of Clinical Nutrition, 2005, vol. 81, p. 341-354.

64. Q. Liu, W. Ju, J.G. Crowston et coll., "Oxidative stress Is an early event in hydrostatic pressure-induced retinal ganglion cell damage », Investigative Ophthalmology \& Visual Science, 2007, vol. 48, p. 4580-4589.

65. S. Mandel, T. Amit, L. Reznichenko et coll., « Green Tea catechins as Brain- 
Permeable, natural iron chelatorsantioxidants for the treatment of neurodegenerative disorders ", Molecular Nutrition \& Food Research, 2006, vol. 50, p. 229-234.

66. B.A. Sutherland, R.M. Rahman et I. Appleton, « Mechanisms of action of green tea chatechins, with a focus on ischemia-Induced neurodegeneration », Journal of Nutritional Biochemistry, 2006, vol. 17, p. 291-306.

67. B. Zhao, « Natural antioxidants for neurodegenerative diseases ", Molecular Neurobiology, 2005, vol. 31, p. 283-293.

68. W.J. Yen, B.S. Wang, L.W. Chang et P. D. Duh, «Antioxidant properties of roasted coffee residues ", Journal of Agricultural and Food Chemistry, 2005, vol. 53, p. 2658-2663.

69. K.B. Miller, D.A. Stuart, N.L. Smith et coll., " Antioxidant activity and polyphenol and procyanidin contents of selected commercially available cocoacontaining and chocolate products in the United States ", Journal of Agricultural and Food Chemistry, 2006, vol. 54, p. 40624068.
70. T. Haufschild, H.J. Kaiser, T. Preisig et coll., « Influence of red wine on visual function and Endothelin-1 plasma level in a patient with optic neuritis ", Annals of Neurology, 2003, vol. 53, p. 825-826.

71. V.L. Singleton et P. Esau, « Phenolic substances in grapes and wine, and their significance », Advances in Food Research (supplément), 1969, vol. 1, p. 1-261.

72. M. Rhone et A. Basu, «Phytochemicals and Age-Related eye diseases ", Nutrition Reviews, 2008, vol. 66, p. 465-472.

73. JoAnn Giaconi, M.D., animatrice, «Nutritional associations with glaucoma among older black women », $18^{\mathrm{e}}$ assemblée annuelle de l'American Glaucoma Society, mars 2008, Washington, D.C.

74. M.N. Feinglos et T.E. Totten, « Are you what you eat, or how much you eat? The case of Type 2 Diabetes Mellitus ", Archives in Internal Medicine, 2008, vol.168, p. 1485-1486.

75. J.H. O’Keefe, N.M. Gheewala et J.O. O'Keefe, « Dietary strategies for improving post-prandial glucose, lipids, inflammation, and cardiovascular health ", Journal of the American College of Cardiology, 2008, vol. 51, p. 249-255.
76. M. Karim, K. McCormick et C.T. Kappagoda, «Effects of cocoa extracts on Endothelium-Dependent relaxation ", Journal of Nutrition, 2000, vol. 130, p. 2105S-2108S.

77. D. Taubert, R. Roesen, C. Lehmann C, et coll., «Effects of low habitual cocoa intake on blood pressure and bioactive nitric oxide: A Randomized Controlled Trial ", Journal of the American Medical Association, 2007, vol. 298, p. 49-60.

78. S.C. Larsson, S. Mannisto, M.J. Virtanen et coll. «Coffee and tea consumption and risk of stroke subtypes in male smokers », Stroke, 2008, vol. 39, p. 16811687.

79. Base de données Cochrane Reviews, accessible en ligne depuis le 18 juillet 2007.

80. J.A. Meyerhardt, D. Niedzwiecki, D. Hollis et coll., « Association of dietary patterns with cancer recurrence and survival in patients with Stage III colon cancer ", Journal of the American Medical Association, 2007, vol. 298, p. 754-764.
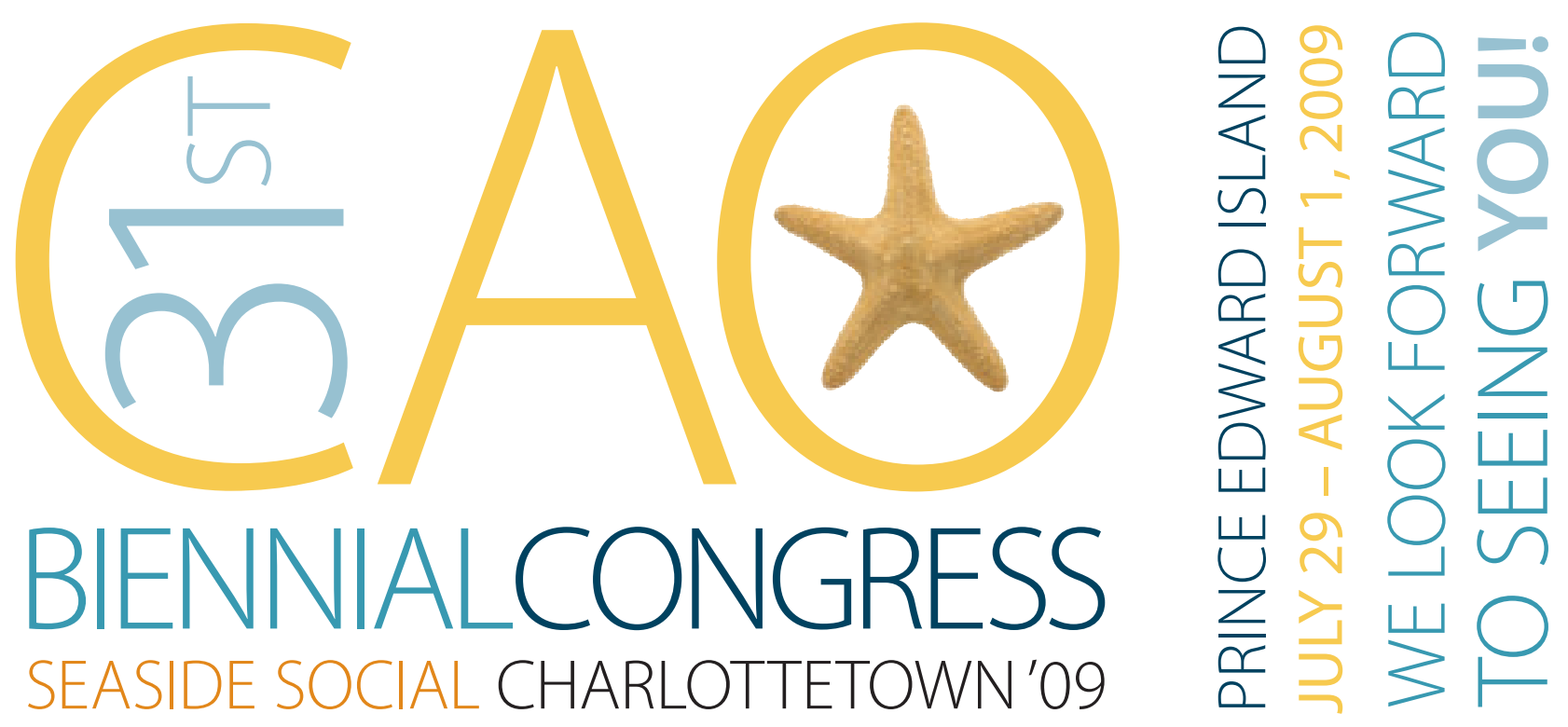